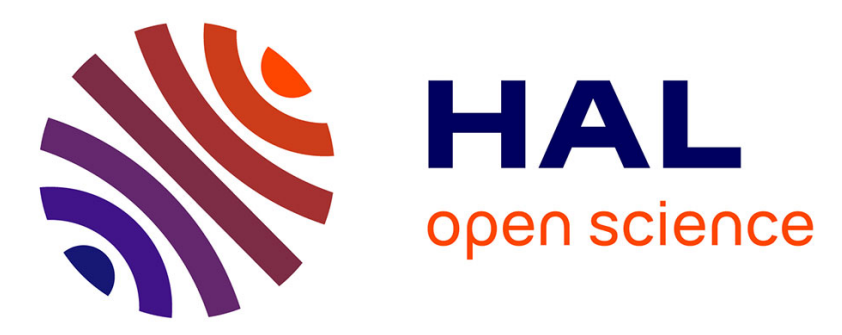

\title{
Joint Analysis of Multiple Datasets by Cross-Cumulant Tensor (Block) Diagonalization
}

\author{
Dana Lahat, Christian Jutten
}

\section{To cite this version:}

Dana Lahat, Christian Jutten. Joint Analysis of Multiple Datasets by Cross-Cumulant Tensor (Block) Diagonalization. SAM 2016 - 9th IEEE Sensor Array and Multichannel Signal Processing Workshop, Jul 2016, Rio de Janeiro, Brazil. hal-01351209

\section{HAL Id: hal-01351209 https://hal.science/hal-01351209}

Submitted on 2 Aug 2016

HAL is a multi-disciplinary open access archive for the deposit and dissemination of scientific research documents, whether they are published or not. The documents may come from teaching and research institutions in France or abroad, or from public or private research centers.
L'archive ouverte pluridisciplinaire HAL, est destinée au dépôt et à la diffusion de documents scientifiques de niveau recherche, publiés ou non, émanant des établissements d'enseignement et de recherche français ou étrangers, des laboratoires publics ou privés. 


\title{
JOINT ANALYSIS OF MULTIPLE DATASETS BY CROSS-CUMULANT TENSOR (BLOCK) DIAGONALIZATION ${ }^{\dagger}$
}

\author{
Dana Lahat and Christian Jutten
}

\author{
GIPSA-Lab, UMR CNRS 5216, Grenoble Campus, BP46, 38402 Saint-Martin-d'Hères, France
}

\begin{abstract}
In this paper, we propose approximate diagonalization of a crosscumulant tensor as a means to achieve independent component analysis (ICA) in several linked datasets. This approach generalizes existing cumulant-based independent vector analysis (IVA). It leads to uniqueness, identifiability and resilience to noise that exceed those in the literature, in certain scenarios. The proposed method can achieve blind identification of underdetermined mixtures when single-dataset cumulant-based methods that use the same order of statistics fall short. In addition, it is possible to analyse more than two datasets in a single tensor factorization. The proposed approach readily extends to independent subspace analysis (ISA), by tensor block-diagonalization. The proposed approach can be used as-is or as an ingredient in various data fusion frameworks, using coupled decompositions. The core idea can be used to generalize existing ICA methods from one dataset to an ensemble.
\end{abstract}

Index Terms - Independent vector analysis; tensor diagonalization; blind source separation; coupled decompositions; data fusion

\section{INTRODUCTION}

In this paper, we propose a cumulant-based approach for the joint analysis of an ensemble of datasets that admit the independent vector analysis (IVA) [1] model. IVA is a framework that addresses an ensemble of independent component analysis (ICA) [2] problems by exploiting not only the statistical independence within each dataset but also dependence among latent sources in different datasets. IVA is further explained in Section 2. An advantage of IVA over analysing each dataset individually is that it aligns the estimated sources in all datasets with the same (arbitrary) permutation, thus obviating the need to resolve the individual arbitrary permutation in each mixture separately. In addition, IVA can generally separate mixtures of Gaussian real stationary sources with spectra identical up to a scaling factor, a problem that does not have a unique solution when each mixture is treated individually. In this paper, we add to the list of useful properties of IVA (i) better resilience to certain types of noise, and (ii) enhanced uniqueness and identifiability in the presence of underdetermined mixtures, for non-Gaussian data.

Most IVA-type methods use cross-correlations, i.e., secondorder cross-cumulants, e.g., [3-6], or a multivariate contrast with a nonlinear function, e.g., $[1,7]$. The idea to explicitly use crosscumulants of higher-order statistics (HOS) for IVA first appeared in $[8,9]$. Their idea is to jointly diagonalize slices of cross-cumulant tensors, where each cross-cumulant involves a pair of datasets. In

This work is supported by the project CHESS, 2012-ERC-AdG-320684. GIPSA-Lab is a partner of the LabEx PERSYVAL-Lab (ANR-11-LABX0025).

${ }^{\dagger}$ This is very close to the official version, published in Proc. SAM 2016. order to jointly process several sets of (cross) cumulants, the factorizations are coupled in a specific way that was termed generalized joint diagonalization (GJD). IVA via GJD can be applied to secondorder statistics (SOS) and up. When fourth-order statistics are used, GJD can be regarded as a multiset counterpart of joint approximate diagonalisation of eigen-matrices (JADE) [10]. The GJD analytical framework in [9] is prewhitening-based and not designed to deal with underdetermined scenarios. When introducing GJD, Li et al. [9] mention that in principle, one may use cross-cumulants taken from more than two datasets. However, they proceed only with pairs of cross-cumulants, explaining that groups of more than two contribute less to the separation than slices involving variables from only two datasets, due to noise and finite sample size. In this paper, we explain, in Section 2, how cross-cumulants that involve $K \geq 2$ different datasets can achieve enhanced resilience to noise. We show how methods based on cross-cumulants enjoy uniqueness and identifiability that exceed those of single-dataset cumulant-based methods that use the same order of statistics. Finally, our approach allows to analyse more than two datasets in a single tensor factorization, as opposed to GJD. For SOS, i.e., $K=2$, GJD coincides with our approach. For $K \geq 3$, our approach is, naturally, not suitable for Gaussian data.

In the following, we denote scalars, vectors, matrices and higher-order arrays (tensors) by $a, \mathbf{a}, \mathbf{A}$ and $\mathcal{A}$, respectively. The $n$th column and $(m, n)$ th entry of $\mathbf{A}$ are denoted by $\mathbf{a}_{n}$ and $a_{m, n}$, respectively. $(\cdot)^{\top}$ and $\|\cdot\|$ denote transpose and the Frobenius norm, respectively. The mode- $n$ product $\times_{n}$ between $\mathcal{C} \in \mathbb{R}^{I_{1} \times \cdots \times I_{N}}$ and $\mathbf{A} \in \mathbb{R}^{J_{n} \times I_{n}}$ yields an $I_{1} \times \cdots \times I_{n-1} \times J_{n} \times I_{n+1} \cdots \times I_{N}$ array whose entries are given by $\left[\mathcal{C} \times{ }_{n} \mathbf{A}\right]_{i_{1}, \ldots, i_{n-1}, j_{n}, i_{n+1}, \ldots, i_{N}}=$ $\sum_{i_{n}=1}^{I_{n}} c_{i_{1}, \ldots, i_{n}, \ldots, i_{N}} a_{j_{n}, i_{n}}$ [11, Definition 4]. The outer product of $N$ non-zero vectors $\mathbf{a}_{n} \in \mathbb{R}^{I_{n} \times 1}$ is a rank-1 $I_{1} \times \cdots \times I_{N}$ tensor $\mathcal{C}=$ $\mathbf{a}_{1} \circ \cdots \circ \mathbf{a}_{N}$ whose entries are given by $c_{i_{1}, \ldots, i_{N}}=a_{1, i_{1}} \cdots a_{N, i_{N}}$.

\section{MODEL AND PROBLEM FORMULATION}

Consider $K$ datasets, each admitting an ICA model,

$$
\mathbf{x}^{[k]}(t)=\mathbf{A}^{[k]} \mathbf{s}^{[k]}(t)+\mathbf{n}^{[k]}(t) \quad 1 \leq t \leq T, 1 \leq k \leq K
$$

where $\mathbf{A}^{[k]} \in \mathbb{K}^{\left[{ }^{[k]} \times R\right.}, \mathbf{x}^{[k]}(t) \in \mathbb{K}^{I^{[k]} \times 1}$ and $\mathbf{s}^{[k]}(t) \in \mathbb{K}^{R \times 1}$ represent a mixing matrix, observations and latent sources, respectively, whose elements are real or complex, $\mathbb{K} \in\{\mathbb{C}, \mathbb{R}\}$. The elements of $\mathbf{s}^{[k]}(t)$ are sampled from random vectors that follow the IVA model. IVA is a generalization of ICA to multiple datasets in which the $K$ elements of random vector $\mathbf{s}_{r}=\left[s_{r}^{[1]}, \ldots, s_{r}^{[K]}\right]^{\top}$, $r=1, \ldots, R$, are statistically dependent whereas the pairs $\left(\mathbf{s}_{r}, \mathbf{s}_{r^{\prime}}\right)$ are statistically independent for any $r \neq r^{\prime}$. The noise random processes $\mathbf{n}^{[k]} \in \mathbb{K}^{[k]} \times 1$ are mutually statistically independent for any $k \neq k^{\prime}$, as well as statistically independent of all sources. 


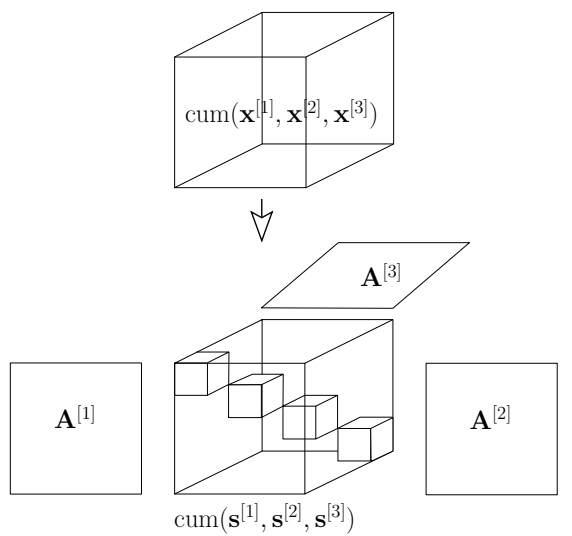

Fig. 1: Diagonalization of a third-order cross-cumulant tensor. In this example, $K=3$ and $R=4$.

Given a set of observations $\mathcal{X}=\left\{\mathbf{x}^{[k]}(t)\right\}_{k=1, t=1}^{K, T}$, the goal of IVA is to separate the sources and/or identify the mixtures by exploiting not only the statistical independence within each set of measurements but also the dependence among sets of measurements. Whenever $\mathbf{A}^{[k]}$ is full column rank, the corresponding samples $\mathbf{s}^{[k]}(t)$ may be estimated by multiplying $\mathbf{x}^{[k]}(t)$ with a left inverse of an estimate of $\mathbf{A}^{[k]}$. Otherwise, the samples cannot be estimated without resorting to additional assumptions (e.g., [12, Chapter 9.2.1]). The latter often occurs when $I^{[k]}<R$, i.e., underdetermined. In this case, one has to suffice with "blind identification", i.e., estimating only $\mathbf{A}^{[k]}$. In this paper, we propose to achieve these inferences by factorizing a cross-cumulant tensor, as we now explain.

For $K \geq 2$, the $K$ th-order $I^{[1]} \times \cdots \times I^{[K]}$ cross-cumulant of $\mathcal{X}$ is given by

$$
\begin{aligned}
\mathcal{C}_{X}^{K} & =\operatorname{cum}\left(\mathbf{x}^{[1]}, \ldots, \mathbf{x}^{[K]}\right) \\
& =\mathcal{C}_{S}^{K} \times{ }_{1} \mathbf{A}^{[1]} \times{ }_{2} \mathbf{A}^{[2]} \times \cdots \times{ }_{K} \mathbf{A}^{[K]}
\end{aligned}
$$

(holds for $K=1$ if the noise has zero mean) where the $K$ th-order $R \times \cdots \times R$ cross-cumulant $\mathcal{C}_{S}^{K}=\operatorname{cum}\left(\mathbf{s}^{[1]}, \ldots, \mathbf{s}^{[K]}\right)$ is diagonal. A key assumption is that the main-diagonal elements do not vanish. The right-hand side (RHS) of (2) is due to the linearity property of cumulants (e.g., [13, Chapter 2]). The noise term vanishes from the cross-cumulants, even for $K=2$, due to its statistical independence properties. We conclude that approximating a sample cross-cumulant of $\mathcal{X}$ with the model in (2) yields estimates of all factors $\mathcal{A}=\left\{\mathbf{A}^{[k]}\right\}_{k=1}^{K}$, with the added benefit of (asymptotically) getting rid of the noise at no additional computational cost. Figure 1 illustrates this idea.

\subsection{Implications}

Equation (2) can be rewritten as

$$
\mathcal{C}_{X}^{K}=\sum_{r=1}^{R} c_{s_{r}}^{K} \mathbf{a}_{r}^{[1]} \circ \mathbf{a}_{r}^{[2]} \circ \cdots \circ \mathbf{a}_{r}^{[K]}
$$

where $c_{s_{r}}^{K}$ denotes the $K$ th-order cumulant of $s_{r}^{[1]}, \ldots, s_{r}^{[K]}, c_{s_{r}}^{K}=$ $\operatorname{cum}\left(s_{r}^{[1]}, \ldots, s_{r}^{[K]}\right)$. Equation (3) rewrites $\mathcal{C}_{X}^{K}$ as a sum of $R$ rank1 terms, where the $r$ th summand represents the contribution of the $r$ th source in all $K$ datasets. For the smallest $R$ for which (3) holds exactly, this factorization amounts to canonical polyadic decomposition (CPD) [14, 15]/parallel factor analysis (PARAFAC) [16]. This fact has the following implications.
Uniqueness: CPD is generically unique under mild conditions (e.g., $[17,18]$ ). Hence, the proposed approach is naturally suitable for underdetermined ICA. Furthermore, the ensemble may admit a unique decomposition even if some of the underdetermined ICA are not identifiable individually with the same order of statistics. We point out that the cumulant-based GJD framework has a similar uniqueness property. The idea that coupled decompositions can provide uniqueness of the ensemble even when each dataset is not individually unique has already been proved in certain types of coupled tensor decompositions [19] and SOS-based IVA [20]. Hence, the proposed approach is another demonstration of the enhanced uniqueness capacities of coupled decompositions.

Computation: Basically, we may optimize (2) and (3) with any general-purpose algorithm that approximates a $K$ th-order tensor with a sum of $R$ rank-1 terms. A similar observation has previously been made with respect to (w.r.t.) underdetermined ICA [12, Chapter 9.4.3]. For a discussion of various aspects of CPD optimization for source separation, see, e.g., [12, Chapter 9.4.3] and references therein. Dedicated tensor diagonalization algorithms such as [21-23] may also be used, whenever the data admits their model assumptions. Constraints such as orthogonality may be imposed as well, e.g., [24]. Since (2) amounts to a Tucker [25] format with a diagonal core, then, in principle, one may use any general-purpose Tucker decomposition algorithm for the optimization. However, uniqueness issues inherent to Tucker-type decompositions may result in difficulty attributing factors to specific sources and hence, weaker interpretability.

\subsection{Generalization to Multidimensional Components}

Until now, we discussed only ICA and rank-1 components. However, all previous results are readily generalizable to rank- $L_{r}^{[k]} \geq 1$ (this variant of IVA is sometimes termed joint independent subspace analysis (JISA) [26, 27]) by replacing the diagonal structure of the tensor with block-diagonal. For example, in the third-order case in Figure 1, the $r$ th block on the diagonal now stands for an $L_{r}^{[1]} \times L_{r}^{[2]} \times L_{r}^{[3]}$ cube instead of a $1 \times 1 \times 1$ scalar. The corresponding factorization is "decomposition in rank- $\left(L_{r}^{[1]}, L_{r}^{[2]}, \ldots, L_{r}^{[K]}\right)$ terms" [28]. Apart from the block term decomposition (BTD) algorithms in, e.g., [29,30], it has been proposed to use tensor diagonalization to uncover the underlying block structure [22,23].

A concluding remark is in order. Whereas ICA has numerous cumulant-based methods, with independent subspace analysis (ISA) the situation is quite different. The first cumulant-based approach to ISA is [31], which proposes to "partially diagonalize" a fourth-order cumulant tensor. A subspace variant of JADE is proposed in [32]. Tichavský et al. [22] mention that tensor block-diagonalization can be applied to cumulant-based ISA; however, they do not develop further this idea.

\section{NUMERICAL VALIDATION}

In this section, we illustrate by numerical examples the capability of the proposed approach to separate sources and identify mixtures in well-posed and underdetermined scenarios.

\subsection{Figure of Merit}

Whenever $\mathbf{A}^{[k]}$ has full column rank, we are interested in the quality of separation of $\mathbf{x}^{[k]}(t)=\sum_{r=1}^{R} \mathbf{x}_{r}^{[k]}(t)$ into $R$ unique rank-1 elements $\mathbf{x}_{r}^{[k]}(t)=\mathbf{a}_{r}^{[k]} s_{r}^{[k]}(t)$. The latter are obtained from the obser- 
vations using $\mathbf{x}_{r}^{[k]}(t)=\mathbf{P}_{r}^{[k]} \mathbf{x}^{[k]}(t)$, where the rank-1 $I^{[k]} \times I^{[k]}$ matrices $\mathbf{P}_{r}^{[k]}$ are oblique projections onto $\operatorname{span}\left(\mathbf{a}_{r}^{[k]}\right)$ orthogonally to all $\operatorname{span}\left(\mathbf{a}_{r^{\prime}}^{[k]}\right), r^{\prime} \neq r$, and can be computed directly from $\mathbf{A}^{[k]}[33]$. Similarly, $\widehat{\mathbf{x}}_{r}^{[k]}(t)=\widehat{\mathbf{P}}_{r}^{[k]} \mathbf{x}^{[k]}(t)$, via $\widehat{\mathbf{A}}^{[k]}$, for their estimated counterparts. We choose to quantify the quality of separation using the normalized empirical mean square error (MSE)

$$
\widehat{\operatorname{MSE}}_{r}^{[k]}=\sum_{t=1}^{T}\left\|\widehat{\mathbf{x}}_{r}^{[k]}(t)-\mathbf{x}_{r}^{[k]}(t)\right\|^{2} / \sum_{t=1}^{T}\left\|\mathbf{x}_{r}^{[k]}(t)\right\|^{2}
$$

The use of scale-invariant projections obviates the need to normalize the source samples in order to find an optimal match with the original samples, in the performance analysis stage. Another motivation is that scale-invariant projections are the natural way to deal with terms of rank larger than one [33], see Section 2.2; scale-dependent objects require normalization, which is not well-defined.

In the underdetermined case, when the sources cannot be separated, we quantify the quality of blind identification, i.e., estimation of each factor $\mathbf{A}^{[k]}$, using the relative error, defined as

$$
\operatorname{RelErr}^{[k]}=\frac{\left\|\mathbf{A}^{[k]}-\widehat{\mathbf{A}}^{[k]}\right\|}{\left\|\mathbf{A}^{[k]}\right\|}
$$

In order to correct the arbitrary permutation and scaling within each estimate $\widehat{\mathbf{A}}^{[k]}$, we use Tensorlab [30]'s function cpderr.

\subsection{Experimental Setup}

We compare our approach with two cumulant-based algorithms, JADE [10] and GJD [9], both are prewhitening-based. We test our proposed cross-cumulant diagonalization approach in two scenarios. In the first, the factors are unconstrained. In the second, full column rank factors are constrained to be orthogonal (there is no added value in orthogonality in underdetermined mixtures [34]). Both scenarios were implemented using CPD-based algorithms, which were applied to the same whitened samples as the other algorithms. This experimental setup allows us to compare the prewhitening-based algorithms, as well as obtain some further insights about the potential of the CPD for this task.

In order to compute the normalized empirical MSE (4) we first have to align the estimated components w.r.t. their true values. As explained in Section 1, for GJD and our proposed approach, only the arbitrary permutation of the $R$ rank-1 elements needs to be found. For JADE, however, we must find this permutation individually for each dataset. A wrong permutation results in significantly higher MSE. In the following examples, since only $K=4$ datasets are involved, we detect this permutation by simple enumeration on all 4 or 4 ! combinations and picking up the best.

Since JADE uses fourth-order cumulants, our experimental setup involves $K=4$ datasets. Each dataset consists of $R=3$ sources and $T=2 \cdot 10^{3}$ sampling points. The entries of $\mathbf{A}^{[k]}$ are drawn independently from the standard normal distribution. We generate non-Gaussian samples that follow the IVA model by defining, for each $r$, two unit-variance independent symmetric Gaussian mixture $(\mathrm{GM})$ processes with peaks centred at $\pm 4 / \sqrt{17}$, and then combining them using $\alpha_{r}^{[k]} \sim U[0,1]$, which is drawn independently at each sample: $s_{r}^{[k]}=\alpha_{r}^{[k]} g_{1, r}+\left(1-\alpha_{r}^{[k]}\right) g_{2, r}$, where $g_{1, r}, g_{2, r} \sim \mathrm{GM}$. The resulting overall random process is normalized to unit variance. In the following numerical experiments, $\mathbf{A}^{[k]}$ are drawn once and kept fixed throughout $M C=200$ Monte Carlo (MC) trials, in which source samples are drawn anew. We

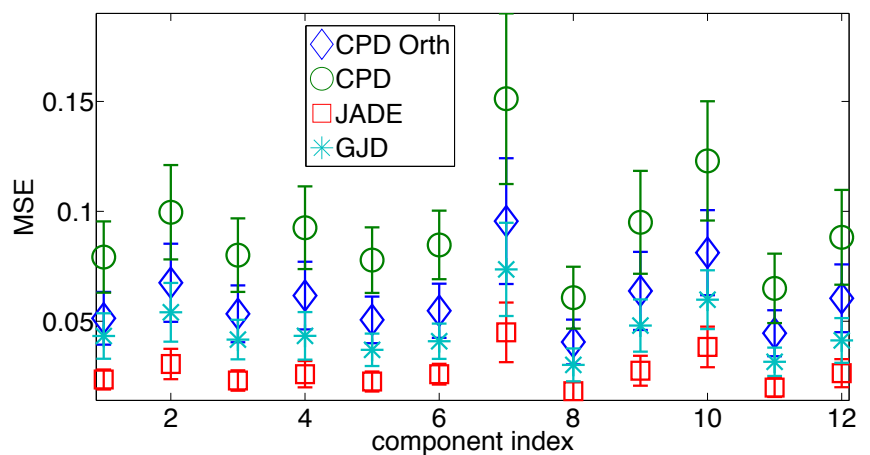

Fig. 2: Normalized MSE in ICA of $K=4$ datasets using fourthorder statistics. In each dataset, $\mathbf{A}^{[k]} \in \mathbb{R}^{3 \times 3}$ and $R=3$ sources. Error bars denote empirical standard deviation. The MSE of component $\mathbf{x}_{r}^{[k]}$ is given at component index $[r+(k-1) R]$.

do not apply additive noise, so estimation error is only due to finite sample size. For JADE and GJD, we use online-available code ${ }^{1}$ with options "N2" and "whole" for GJD. For CPD, we use a Tensorlab [30] implementation with TolFun $=10^{-12}$, TolX $=10^{-12}$. The unconstrained CPD is optimized using cpd_nls, where, for each MC trial, we take the best out of 25 random initializations. CPD with orthogonal factors is optimized using sdf_minf via struct_orth, where the factors are initialized with random values (only once per trial).

1st experiment: Source separation in invertible mixtures. In a first step, we validate proper functionality of our proposed approach in a basic setup of invertible $\mathbf{A}^{[k]} \in \mathbb{R}^{R \times R} \forall k$. Figure 2 depicts the normalized empirical MSE in the estimation of each component. For each pair $(k, r)$, we removed trials for which at least one type of algorithm did not achieve separation ${ }^{2}$. In this experiment, two trials, which corresponds to component \#7 in Figure 2, were removed due to large error in unconstrained CPD.

2nd experiment: blind identification. In a second step, we present the capability of the proposed method to achieve blind identification of underdetermined mixtures. Consider the case of $\mathbf{A}^{[k]} \in \mathbb{R}^{2 \times 3}, k=1,2,3$. By straightforward counting of degrees of freedom, one can verify that ICA with $R=3$ sources and $I^{[k]}=2$ detectors is generally not unique and not identifiable if one exploits only statistics up to order four and all data is in the real domain (e.g., [35, 36]). With our approach, however, this is possible. We guarantee the uniqueness of the CPD of our fourth-order cross-cumulant tensor by setting $\mathbf{A}^{[4]} \in \mathbb{R}^{3 \times 3}$ [18]. Hence, this experiment consists of three underdetermined ICA that cannot be identified by any fourth-order method and one ICA that can be solved by ordinary $\mathrm{JADE}^{3}$. The GJD algorithm [9] is not suitable for datasets with underdetermined mixtures and is thus excluded from this experiment. JADE can be applied only to the fourth dataset. The relative error (5) for all $K=4$ factors using CPD is depicted in Figure 3. The normalized empirical MSE in the estimation of all rank-1 components in the 4th dataset using JADE and CPD is given in Figure 4.

\footnotetext{
${ }^{1}$ http://perso.telecom-paristech.fr/ cardoso/Algo/Jade/jadeR.m, http://mlsp.umbc.edu/codes/jbss_cum4.m

${ }^{2}$ With certain draws of $\mathbf{A}^{[k]}$, both orthogonal and unconstrained CPD did often not converge to a desired solution, regardless of initialization. This matter deserves to be further looked into. For the examples in this paper, only data with no estimation issues were selected.

${ }^{3}$ In fact, stronger uniqueness is generally possible. For example, there are enough degrees of freedom already at two underdetermined mixtures and fourth-order statistics. In addition, a mixing matrix that can be estimated individually may be regarded as a known factor in the ensemble.
} 


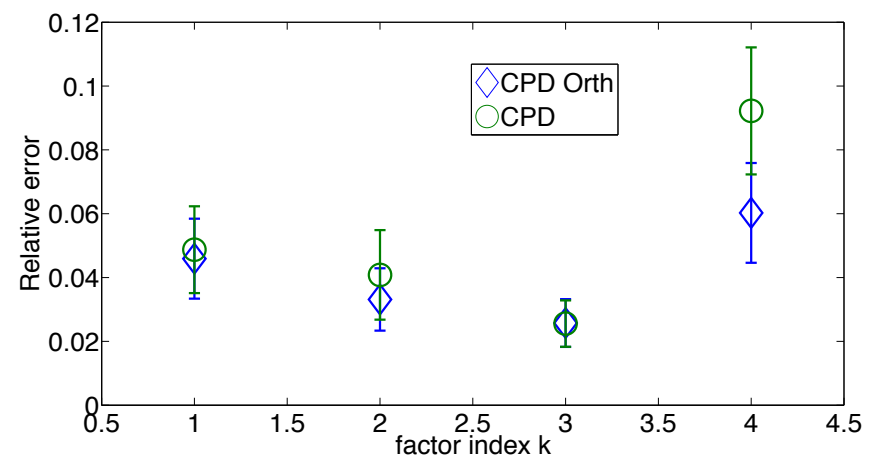

Fig. 3: Relative error in estimating $\mathbf{A}^{[k]}$ in $K=4$ datasets, each with $R=3$ sources. $\mathbf{A}^{[k=1,2,3]} \in \mathbb{R}^{2 \times 3}$. $\mathbf{A}^{[4]} \in \mathbb{R}^{3 \times 3}$. Error bars denote empirical standard deviation.

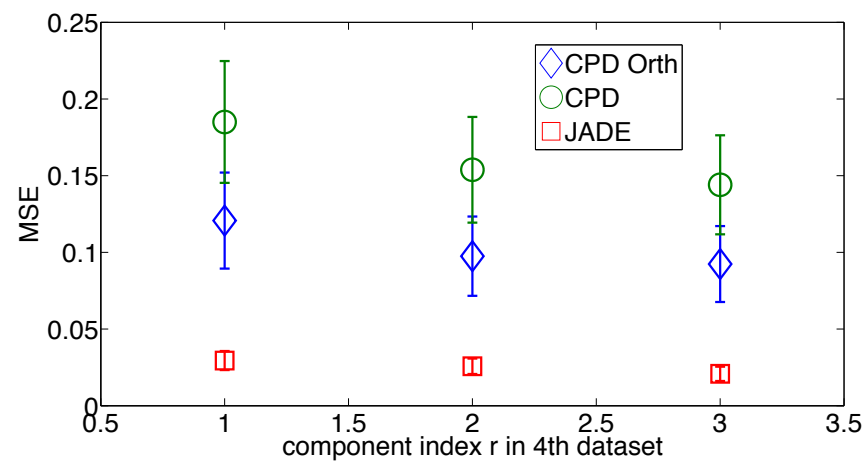

Fig. 4: Normalized MSE in ICA of $K=4$ datasets, each with $R=3$ sources. $\mathbf{A}^{[k=1,2,3]} \in \mathbb{R}^{2 \times 3}$. $\mathbf{A}^{[4]} \in \mathbb{R}^{3 \times 3}$. Here, we depict the MSE only for $k=4$.

\subsection{Discussion of Numerical Results}

The small values of MSE and relative error in Figures 2, 3 and 4 imply successful separation and blind identification. JADE is the algorithm that makes an overall most efficient use of the fourth-order statistics within each dataset and indeed, it achieves the best MSE. The fact that GJD performs worse than JADE deserves to be further looked into. We point out that $[8,9]$ did not compare with JADE. Orthogonally-constrained CPD consistently performs better than the unconstrained CPD, and pretty close to GJD. Given that CPD uses less statistical information than GJD, this is an encouraging indication to the usefulness of the proposed approach. The rather poor performance of unconstrained CPD cannot be explained just by the difference between non-orthogonal vs. prewhitening-based methods. In fact, when testing unconstrained CPD on non-prewhitened samples, its performance was even worse. A possible explanation may be the larger number of variables to estimate in unconstrained factors, compared with fewer ones when orthogonality is imposed. In addition, the cost function of CPD is different than directly minimizing off-diagonal values, a matter than may be more significant in large sample perturbation. Other algorithms (Section 2.1) may perform better. Therefore, although unconstrained CPD achieves the smallest residual error in estimating the original cumulant tensor, it may not be the best candidate for our approach compared with other tensor diagonalization methods. This matter deserves to be further looked into. The fact that orthogonality constraints are void in the underdetermined scenario explains why the relative error is similar for both types of CPD in the first three factors in Figure 3. The performance of the proposed approach may be enhanced by coupling with SOS [10, Sec. 4.2] or with other (cross-) cumulants that carry complementary information. It is interesting to observe where the trade-off between different types of combinations of statistics is, both in terms of error and in computational cost.

The overall conclusion from these experiments is that our paradigm is correct, and that our cross-cumulant tensor diagonalization approach indeed successfully exploits statistical links among datasets to achieve blind identification of underdetermined mixtures even in "adverse" scenarios, when the order of statistics is limited and individual ICA is impossible. However, for more conclusive statements about the usefulness of our proposed approach w.r.t. state of the art, these preliminary numerical results serve only as a proof of concept, and require further theoretical and experimental support.

\section{CONCLUSION}

In this paper, we have shown that factorizing a cross-cumulant tensor of several datasets can achieve simultaneous ICA of all involved mixtures, if certain conditions hold. We explained how cumulant-based IVA may achieve uniqueness of the ensemble that exceeds that of individual underlying datasets, extending previous results on SOS-IVA. We have shown analytically that using crosscumulants provides, asymptotically, resilience to dataset-specific noise. The proposed approach relies directly on the strong uniqueness of CPD/BTD. Even stronger uniqueness, identifiability and interpretability may be achieved by coupling several arrays, adding types of diversity (e.g., nonstationarity), various assumptions and constraints within and among datasets. The proposed approach motivates multiset variants of tensor-based ICA methods such as JADE, its higher-order generalizations [37,38], or methods based on the characteristic function [39] to use this cross-cumulant concept to achieve stronger uniqueness.

\section{REFERENCES}

[1] T. Kim, T. Eltoft, and T.-W. Lee, "Independent vector analysis: An extension of ICA to multivariate components," in Independent Component Analysis and Blind Signal Separation, ser. LNCS, vol. 3889. Springer Berlin Heidelberg, 2006, pp. $165-172$.

[2] P. Comon, "Independent component analysis," in Proc. Int. Signal Process. Workshop on HOS, Chamrousse, France, Jul. 1991, pp. 111-120,

[3] H. Hotelling, "Relations between two sets of variates," Biometrika, vol. 28, no. 3/4, pp. 321-377, Dec. 1936.

[4] J. Kettenring, "Canonical analysis of several sets of variables," Biometrika, vol. 58, no. 3, pp. 433-451, 1971.

[5] Y.-O. Li, T. Adalı, W. Wang, and V. D. Calhoun, "Joint blind source separation by multiset canonical correlation analysis," IEEE Trans. Signal Process., vol. 57, no. 10, pp. 3918-3929, Oct. 2009.

[6] M. Anderson, T. Adalı, and X.-L. Li, "Joint blind source separation with multivariate Gaussian model: Algorithms and performance analysis," IEEE Trans. Signal Process., vol. 60, no. 4, pp. 1672-1683, Apr. 2012.

[7] M. Anderson, G.-S. Fu, R. Phlypo, and T. Adalı, "Independent vector analysis, the Kotz distribution, and performance 
bounds," in Proc. ICASSP, Vancouver, Canada, May 2013, pp. 3243-3247.

[8] X.-L. Li, M. Anderson, and T. Adal1, "Second and higher-order correlation analysis of multiple multidimensional variables by joint diagonalization," in Latent Variable Analysis and Signal Separation, ser. LNCS, vol. 6365. Heidelberg: Springer, 2010, pp. 197-204.

[9] X.-L. Li, T. Adal1, and M. Anderson, "Joint blind source separation by generalized joint diagonalization of cumulant matrices," Signal Process., vol. 91, no. 10, pp. 2314-2322, Oct. 2011.

[10] J.-F. Cardoso and A. Souloumiac, "Blind beamforming for non-Gaussian signals," Radar and Signal Process., IEE Proceedings F, vol. 140, no. 6, pp. 362-370, Dec. 1993.

[11] L. De Lathauwer, B. De Moor, and J. Vandewalle, "Blind source separation by higher-order singular value decomposition," in Proc. EUSIPCO, vol. 1, Edinburgh, Scotland, UK, Sep. 13-16 1994, pp. 175-178.

[12] P. Comon and C. Jutten, Eds., Handbook of Blind Source Separation: Independent Component Analysis and Applications, 1st ed. Academic Press, Feb. 2010.

[13] D. R. Brillinger, Time Series, Data Analysis and Theory. San Francisco, CA: Holden-Day, 1981.

[14] F. L. Hitchcock, "The expression of a tensor or a polyadic as a sum of products," J. Math. Phys., vol. 6, no. 1, pp. 164-189, 1927.

[15] J. D. Carroll and J.-J. Chang, "Analysis of individual differences in multidimensional scaling via an $N$-way generalization of "Eckart-Young" decomposition," Psychometrika, vol. 35, no. 3, pp. 283-319, Sep. 1970.

[16] R. A. Harshman, "Foundations of the PARAFAC procedure: models and conditions for an "explanatory" multimodal factor analysis," UCLA Working Papers in Phonetics, vol. 16, pp. 184, Dec. 1970

[17] J. B. Kruskal, "Three-way arrays: rank and uniqueness of trilinear decompositions, with application to arithmetic complexity and statistics," Linear Algebra Appl., vol. 18, no. 2, pp. 95-138, 1977.

[18] N. D. Sidiropoulos and R. Bro, "On the uniqueness of multilinear decomposition of $N$-way arrays," J. Chemometrics, vol. 14, no. 3, pp. 229-239, May-Jun. 2000.

[19] M. Sørensen and L. De Lathauwer, "Coupled canonical polyadic decompositions and (coupled) decompositions in multilinear rank- $\left(L_{r, n}, L_{r, n}, 1\right)$ terms-part I: Uniqueness," SIAM J. Matrix Anal. Appl., vol. 36, no. 2, pp. 496-522, Apr. 2015.

[20] J. Vía, M. Anderson, X.-L. Li, and T. Adalı, "Joint blind source separation from second-order statistics: Necessary and sufficient identifiability conditions," in Proc. ICASSP, Prague, Czech Republic, May 2011, pp. 2520-2523.

[21] M. Sørensen, P. Comon, S. Icart, and L. Deneire, "Approximate tensor diagonalization by invertible transforms," in Proc. EUSIPCO, Glasgow, Scotland, UK, Aug. 2009, pp. 500-504.

[22] P. Tichavský, A. H. Phan, and A. Cichocki, "Non-orthogonal tensor diagonalization, a tool for block tensor decompositions," arXiv:1402.1673 [cs.NA], 2015.

[23] Y. Liu, X.-F. Gong, and Q. Lin, "Non-orthogonal tensor diagonalization based on successive rotations and LU decomposition," in Proc. ICNC, Zhangjiajie, China, Aug. 2015, pp. 102107.
[24] M. Sørensen, L. De Lathauwer, P. Comon, S. Icart, and L. Deneire, "Canonical polyadic decomposition with a columnwise orthonormal factor matrix," SIAM J. Matrix Anal. Appl., vol. 33, no. 4, pp. 1190-1213, 2012.

[25] L. R. Tucker, Contributions to Mathematical Psychology. New York: Holt, Rinehardt \& Winston, 1964, ch. The extension of factor analysis to three-dimensional matrices, pp. 109127.

[26] D. Lahat and C. Jutten, "Joint independent subspace analysis using second-order statistics," IEEE Trans. Signal Process., vol. 64, no. 18, pp. 4891-4904, Sep. 2016.

[27] R. F. Silva, S. Plis, T. Adalı, and V. D. Calhoun, "Multidataset independent subspace analysis extends independent vector analysis," in Proc. ICIP, Paris, France, Oct. 2014, pp. 2864-2868.

[28] L. De Lathauwer, "Decompositions of a higher-order tensor in block terms. Part II: Definitions and uniqueness," SIAM J. Matrix Anal. Appl., vol. 30, no. 3, pp. 1033-1066, 2008.

[29] L. De Lathauwer and D. Nion, "Decompositions of a higherorder tensor in block terms. Part III: Alternating least squares algorithms," SIAM J. Matrix Anal. Appl., vol. 30, no. 3, pp. 1067-1083, 2008.

[30] L. Sorber, M. Van Barel, and L. De Lathauwer, "Tensorlab v2.0," Jan. 2014. [Online]. Available: http://www.tensorlab. net/

[31] L. De Lathauwer, B. De Moor, and J. Vandewalle, "Fetal electrocardiogram extraction by source subspace separation," in Proc. IEEE SP/ATHOS Workshop on HOS, Girona, Spain, Jun. 1995, pp. 134-138.

[32] F. J. Theis, "Towards a general independent subspace analysis," in Proc. NIPS, 2007, pp. 1361-1368.

[33] J.-F. Cardoso, "Multidimensional independent component analysis," in Proc. ICASSP, vol. 4, Seattle, WA, May 1998, pp. 1941-1944.

[34] L. De Lathauwer, "Simultaneous matrix diagonalization: the overcomplete case," in Proc. ICA, vol. 8122, Nara, Japan, Apr. 2003, pp. 821-825.

[35] P. Comon, "Tensor diagonalization, a useful tool in signal processing," in Proc. IFAC-SYSID, vol. 1, Copenhagen, Denmark, Jul. 1994, pp. 77-82.

[36] L. De Lathauwer, P. Comon, B. De Moor, and J. Vandewalle, "ICA algorithms for 3 sources and 2 sensors," in Proc. IEEE Sig. Process. Workshop on HOS, Caesarea, Israel, Jun. 1999, pp. 116-120.

[37] L. De Lathauwer, B. De Moor, and J. Vandewalle, "Independent component analysis and (simultaneous) third-order tensor diagonalization," IEEE Trans. Signal Process., vol. 49, no. 10, pp. 2262-2271, 2001.

[38] E. Moreau, "A generalization of joint-diagonalization criteria for source separation," IEEE Trans. Signal Process., vol. 49, no. 3, pp. 530-541, Mar 2001.

[39] P. Comon and M. Rajih, "Blind identification of underdetermined mixtures based on the characteristic function," Signal Process., vol. 86, no. 9, pp. 2271-2281, Sep. 2006, special Section: Signal Processing in UWB Communications. 